\title{
Childhood Atypical Teratoid/Rhabdoid Tumor
}

National Cancer Institute

\section{Source}

National Cancer Institute. Childhood Atypical Teratoid/Rhabdoid Tumor. NCI Thesaurus.

Code C68634.

An atypical teratoid/rhabdoid tumor occurring in children. 